\title{
Linx
}

Revue des linguistes de l'université Paris X Nanterre

$77 \mid 2018$

Regards croisés sur le futur en français et dans différentes langues romanes

\section{Regards philosophiques et linguistiques sur le signifié de langue du futur de l'indicatif dans les langues romanes}

\section{Sophie Azzopardi}

\section{OpenEdition}

\section{Journals}

Édition électronique

URL : http://journals.openedition.org/linx/2697

DOI : $10.4000 /$ linx.2697

ISSN : 2118-9692

\section{Éditeur}

Presses universitaires de Paris Nanterre

Édition imprimée

Date de publication : 30 octobre 2018

Pagination : 17-40

ISBN : 978-2-84016-329-9

ISSN : 0246-8743

\section{Référence électronique}

Sophie Azzopardi, «Regards philosophiques et linguistiques sur le signifié de langue du futur de

l'indicatif dans les langues romanes », Linx [En ligne], 77 | 2018, mis en ligne le 30 avril 2019, consulté le 03 mai 2019. URL : http://journals.openedition.org/linx/2697 ; DOI : 10.4000/linx.2697

Ce document a été généré automatiquement le 3 mai 2019.

Département de Sciences du langage, Université Paris Ouest 


\title{
Regards philosophiques et linguistiques sur le signifié de langue du futur de l'indicatif dans les langues romanes
}

\author{
Sophie Azzopardi
}

\section{Introduction}

1 Le futur dans les langues romanes est un des temps verbaux qui fait l'objet de recherches sans cesse renouvelées pour plusieurs raisons. D'une part parce qu'il situe l'événement dans l'époque future, époque en lien étroit avec la modalité épistémique du possible, ce qui pose le problème de la valeur en langue de ce temps (au croisement de la temporalité et de la modalité) et de son articulation avec les différents emplois qu'il peut avoir en discours ; d'autre part parce qu'il est issu de la grammaticalisation d'une périphrase aspectuelle (infinitif + habere au présent) et qu'il est actuellement concurrencé par une périphrase composée d'un verbe de mouvement et de l'infinitif, ce qui soulève la question de l'alternance entre la forme synthétique («futur morphologique») et la forme analytique («futur périphrastique ») et de l'avenir de la forme synthétique actuelle. Par conséquent, les questions soulevées sont principalement de deux ordres :

- des questions d'ordre à la fois philosophique et linguistique autour de la valeur en langue de ce temps (au croisement de la temporalité et de la modalité) et de son articulation avec les différents emplois qu'il peut avoir en discours ;

- des questions autour de l'alternance entre le futur morphologique et le futur périphrastique et de l'avenir de la forme synthétique actuelle.

2 Ces questions sont transversales et sont abordées depuis différentes perspectives : synchronique (Martin, 1981; Bahur, 1992; Álvarez Castro, 2007; Bres, 2009; Abouda, Azzopardi, Skrovec, 2016 i.a.), diachronique (Fleischman, 1982 ; Bybee, Perkins, Pagliuca, 1994 ; Vetters, Lière, 2009 ; Bres, Labeau, 2012 i.a.), contrastive (Rocci, 2000 ; Squartini, 2004 ; Barceló, 2007; Siletti, 2009; Azzopardi, 2011 ; Baranzini 2017; Ricci 2017 i.a.) , 
acquisitionnelle (Sabeau-Jouannet, 1973; Morgenstern, Parisse, Sekali, 2010 i.a.) ou encore didactique (Péret, 2013 i.a.). L'objectif dans le cadre de cet article est de mener une réflexion d'ordre plus théorique compatible avec les différentes perspectives énoncées supra. Il s'agira de donner des éléments d'analyse descriptive du signifié de la valeur en langue du futur qui permette, en diachronie et en synchronie :

(a) de rendre compte des spécificités de ce temps en termes de temporalité et de modalité ;

(b) de rendre compte des différents emplois en discours du futur ;

(c) d'analyser la concurrence entre futur morphologique et futur périphrastique ;

(d) de mettre en évidence les points communs et différences entre les différentes

langues romanes.

Pour ce faire, on abordera dans un premier temps le futur dans une perspective ontologique et linguistique (section 1), ce qui permettra de poser les fondements conceptuels de la valeur en langue du futur qui sera développée dans ce travail. On décrira ensuite le système de modélisation des temps verbaux de l'indicatif dans le cadre duquel l'analyse du futur est menée (section 2) avant de décrire la valeur en langue des différentes formes de futur (section 3). Cette valeur en langue sera enfin mise à l'épreuve de l'analyse dans la dernière partie (section 4) qui sera consacrée à l'étude du fonctionnement du futur dans différents emplois en discours.

\section{Cadres théoriques : perspective ontologique et linguistique}

La conception et l'expérience que les sujets ont du temps est en lien étroit avec l'expression linguistique qu'ils en ont. Un des problèmes auxquels se trouve confronté le linguiste à l'heure d'analyser le fonctionnement des tiroirs verbaux, et a fortiori celui du futur et du conditionnel, réside justement dans cette imbrication entre langage et pensée, entre temps phénoménologique et temps linguistique. Il s'agira ici de présenter les principaux enjeux de la conception philosophique et socio-culturelle du temps et de l'avenir dans une perspective diachronique dans le but de mettre en relation la valeur en langue du futur et les moyens linguistiques utilisés pour situer un événement dans l'avenir avec la représentation que les sujets ont de cette partie du temps.

\subsection{De l'Antiquité à Saint-Augustin : la conception mythique}

La nécessité pour l'Homme de se situer dans l'espace et dans le temps est présente dès l'époque archaïque. La géographie spatio-temporelle mythique s'organise autour de deux axes, l'espace et le temps, qui se rejoignent en un point central. Ce point central représente le centre du monde (Boskovic, $2006: 3$ ). C'est le seul point fixe qui permet à l'Homme de se situer et de se repérer. Le centre du monde est également le lieu de jonction de deux univers : le profane et le sacré. Le temps profane correspond à celui qui est expérimenté par l'Homme, à sa vie et à son espace. Il est linéaire et irréversible. Le temps sacré quant à lui est cyclique, réversible et se voit représenté par un espace circulaire : c'est celui des rites et du récit des origines, transmis par les ancêtres. Sa fonction est d'organiser le temps profane, de le protéger du chaos par le biais de la répétition et de l'identité des différents espaces temporels : le passé est rejoué dans le présent et sert à son tour de modèle pour créer ce qui est à venir : 
Les mythes et les rites cosmogoniques sont à l'origine de tout savoir ; ils représentent à la fois les modèles de la création divine et les matrices de toute sa création postérieure. (Boskovic, $2006: 3$ ) pensée judéo-chrétienne qui accorde une place plus importante à l'Homme et marque ainsi le passage d'un temps cyclique calqué sur les rythmes de la nature à un temps linéaire dont le début est marqué par la naissance du Christ. Ce point zéro est également le point de jonction de deux types de temps : le temps éternel de Dieu et le temps historique de l'Homme. Ce qui se situe avant ce point représente le paradis perdu, qui est aussi ce vers quoi l'Homme tend : un retour au paradis et à l'éternité. Cependant, le temps n'est plus cyclique en dépit de l'idée de retour au temps éternel, il a un début et une fin. Ce qui précède la naissance du Christ est compté à rebours, et ce qui est situé après apparaît successivement comme un vecteur matérialisant ce vers quoi l'Homme doit tendre : il doit gagner le paradis, et non y revenir.

C'est cette symétrie et ces trois moments du temps qu'on retrouve chez Saint Augustin qui introduit la dimension psychologique du temps. Pour Saint Augustin en effet, l'avenir n'a pas d'existence en soi et ne peut exister que par l'image projetée qui en est faite dans le présent (Saint Augustin, XI XVIII 24) :

Ainsi, l'avenir n'est pas encore ; s'il n'est pas encore, il n'est pas ; s'il n'est pas, il ne peut se voir ; mais il peut se prédire d'après les choses présentes qui sont déjà et que l'on peut voir.

12 L'avenir est ainsi vu comme une préméditation (XI XVIII 23) :

Mais ce que je sais, c'est que d'ordinaire nous préméditons nos actions futures; que cette préméditation est présente, mais que l'action préméditée n'est pas encore, parce qu'elle est future.

Le temps se révèle donc être un élément subjectif qui n'a pas d'existence en dehors de la conscience que le sujet en a tout en restant dépendant de l'existence de Dieu. La 
conception cognitive du temps émerge avec Saint Augustin et c'est cette prise de conscience du lien entre temps et subjectivité qui sera à la base de la plupart des théories qui seront élaborées par la suite.

Chez Descartes, la dimension cognitive de la conception du temps occupe une place beaucoup plus importante et apporte un éclairage nouveau sur les notions de doute et de certitude et leur lien avec le temps et l'avenir. Descartes n'envisage pas le temps sous l'angle de la subjectivité, mais plutôt comme un phénomène du monde. Le seul instant qui est empreint de certitude, c'est l'instant où survient la pensée. Le passé comme l'avenir sont des constructions mentales et en ce sens, elles sont soumises au doute. Cependant, Descartes met en évidence le fait que chacun de ces instants indubitables peut être transposé à un autre moment du temps, envisagé séparément de l'instant présent et avec autant de certitude.

Du point de vue socio-culturel, ce qui intervient avec la science cartésienne, c'est le fait que l'Homme devient maître de la nature et que le développement des connaissances et des techniques va permettre de modifier le cours des choses. De là découle que le futur devient prévisible. On peut le voir, ou du moins l'entrevoir, parce qu'il peut être construit par le biais des actions accomplies par l'Homme, dans le cadre de ce que la nature (ou Dieu) détermine comme étant possible.

Avec la conception cognitive, le temps est un temps linéaire ne pouvant émerger qu'à travers la conscience et la subjectivité de l'Homme. La représentation linéaire du temps se fait sous la forme d'un vecteur bidirectionnel et se caractérise par une symétrie entre le passé et le futur séparés par le présent, qui est le seul instant dont on ne peut douter. Le passé comme le futur ne sont pas inscrits dans la réalité et sont des constructions mentales du sujet. De ce fait, ils reçoivent le même degré de certitude. On peut douter du passé comme de l'avenir parce qu'ils ne sont que des images mentales, mais ils sont certains, l'un parce qu'il a déjà été, et l'autre parce qu'il est prévisible, dans le cadre de ce que la nature ou Dieu permet, parce que l'Homme a un certain savoir.

Lorsque l'on croise ce regard philosophique avec un point de vue sur l'expression linguistique du futur dans les langues romanes, la forme employée à ces différentes époques est majoritairement la forme synthétique issue de la périphrase cantare habeo. On remarquera que ce processus de grammaticalisation de la périphrase s'accompagne d'une modification de sa valeur qui passe d'une valeur d'obligation à une valeur temporelle. Fleischman (1982 : 75) montre que les formes temporelles ont tendance à être synthétiques alors que les formes modales ont tendance à être analytiques :

forms expressing primary temporality tend to be synthetic

forms expressing primary modality tend to be analytic

From a diachronic perspective this hypothesis implies that as modal periphrases become temporalized [...] they are likely in the process to agglutinate [...]. This occurred in the case of chantrai/cantaré, whereas cantar-[pron]-he [...] perished. he de cantar [...] retained its modality and likewise its analytic structure. The importance of grammaticization as a factor in the morphosyntax of this development should not be underestimated. The consolidation of cantare habeo $\rightarrow$ chanterai as a tense functor, entailing a predictable opacity with respect to its obligative origin, is reflected morphologically in the tighter fusion of infinitive + auxiliary than in the case of the results of habeo cantare, where the more transparent semantic structure translates morphologically into a greater independence between the verbal elements. 

d'évolution, et par conséquent de grammaticalisation, de la forme analytique en latin. On distinguera trois étapes : une en latin classique, une en latin tardif et une dernière dans les langues romanes. quod/quid + verbe conjugué, en remplacement de la proposition infinitive :

- Lorsque le verbe principal est au présent, trois formes peuvent être employées pour dénoter un événement situé dans l'ultériorité par rapport au présent : cantabo / cantaturus sum / cantare habeo.

- Lorsque le verbe principal est au passé, seules deux formes peuvent être employées pour dénoter un événement situé dans l'ultériorité par rapport à ce repère passé : cantaturus eram / cantare habebam.

21 Il y avait donc deux structures parallèles, mais dans lesquelles la forme cantabo n'avait pas de correspondant dans le cas d'un verbe principal au passé. Cette forme a par la suite disparu, au même titre que la forme en -urus et les seules formes qui se sont maintenues dans ce type de structure étaient cantare habeo / cantare habebam.

Dans le passage aux langues romanes, suivant le schéma évoqué par Fleischman (1982 : 75), les formes périphrastiques, fréquemment employées pour situer un événement dans l'ultériorité par rapport au verbe principal, se synthétisent du fait de leur emploi principalement dans des énoncés à sens temporel.

\subsection{La période contemporaine : la conception phénoménologique}

La conception phénoménologique du temps se développe en étroite relation avec l'évolution de la science et des techniques à partir de la fin du XIXe siècle. On a mis en évidence le fait que la conception cognitiviste envisage la nature comme un cadre régulateur et fini dans lequel l'Homme peut se mouvoir et agir. Les avancées dans les domaines scientifiques et techniques conduisent l'Homme à appréhender le monde comme une réalité beaucoup plus complexe qu'auparavant, et la découverte de la relativité de certaines lois physiques induit une modification importante de la conception du temps et de l'avenir. L'Homme ne se situe plus dans un cadre fini mais dans un environnement infini. C'est ce que Berger (1964 : 231-232) formule de la façon suivante :

L'homme y [dans le monde contemporain] effectue des actes irréversibles. Il est devenu assez puissant pour altérer définitivement certains équilibres naturels. [...] Parfois encore, il sent qu'il prolonge la nature et qu'il réalise ce que, sans lui, elle n'aurait pu accomplir. [...] Nos moyens de prévoir se sont considérablement accrus. [...] Mais notre science même nous montre que tout est plus complexe que nous ne l'imaginions. [...] l'avenir que nos prévisions semblaient serrer de près devient de plus en plus imprévisible. [...] Il nous faut décidément abandonner l'idée que le monde aurait une problématique finie.

On voit se dessiner tout le paradoxe qui mène de la science positiviste à la conception phénoménologique du temps. La connaissance plus avancée des phénomènes naturels par l'Homme a pour effet de lui révéler un monde autrement plus compliqué que ce qu'il imaginait et du même coup rompt les cadres fixes et relativise les principes qu'il tente 
d'établir par le biais de cette même connaissance. La conséquence de l'abandon de la notion de finitude du monde se traduit par l'irruption de l'idée que l'Homme jouit d'une infinie liberté (Berger, 2006 : 233) :

L'invention bouleverse à une vitesse croissante nos habitudes et nos conditions d'existence. [...] Il nous faut alors dépasser la conception trop étroite de la prévision positiviste, qui se contentait de prolonger le passé dans l'avenir. Demain ne sera pas comme hier. Il sera nouveau et il dépendra de nous. Il est moins à découvrir qu'à inventer. L'avenir de l'homme antique devait être révélé. Celui du savant d'hier pouvait être prévu. Le nôtre est à construire - par l'invention et par le travail.

L'idée de liberté est celle qui traverse la plupart des conceptions contemporaines du temps et de l'avenir de Bergson à Jankélévitch. Chez Jankélévitch, l'avenir et la conscience du temps sont une manifestation de la liberté de l'homme. Pour lui, le temps est un flux irréversible qui est à la fois signe de l'impuissance de l'homme, puisqu'il l'entraîne vers sa mort, et signe de sa puissance. C'est ce qu'il exprime de la façon suivante alors qu'il est invité à réagir à propos du film «La Machine à explorer le temps " de George Pal (1960) lors d'une émission de radio en 1975 :

L'avenir est à la fois mon impuissance et ma puissance. Mon impuissance parce qu'il m'emporte vers la mort, irréversiblement, et ma puissance parce que c'est la seule dimension dans laquelle je puisse agir, vers l'avenir. Sur le passé je ne puis rien, parce qu'il est révolu [...]. Mais sur l'avenir, par contre, tout est possible à la liberté, tout est possible à un homme qui le veut. C'est la dimension de mon pouvoir. ${ }^{1}$

Ainsi, ce qui ressort des diverses conceptions qui ont ici été regroupées sous la dénomination de «conception phénoménologique » du temps, c'est que le temps n'a pas d'existence sans conscience et qu'il est le signe de la liberté créatrice de l'Homme qui dans son présent vécu a le pouvoir de construire à la fois son passé et son avenir. L'idée de possible contenue dans cette définition de l'avenir peut conduire à penser l'avenir comme un temps ramifié, puisque les possibilités sont infinies.

C'est d'ailleurs la conception développée dans les approches logiques du temps notamment celles de Kripke (1963), de Rescher \& Urquhart (1971), et de Gardies (1975) qui se situent à la croisée des perspectives philosophiques et linguistiques sur le temps. Pour Gardies (1975), l'époque passée se définit comme celle d'un temps unilinéaire en ce sens que pour un même sujet, il ne peut y avoir qu'un seul procès qui ait eu lieu à un point donné du temps. L'époque passée est celle de l'irrévocabilité des faits, et on ne peut en changer le cours. En revanche, l'époque future contient une part d'incertitude inhérente à la méconnaissance que l'on a de l'avenir : un sujet peut projeter un procès dans l'avenir, mais tant qu'il ne s'est pas produit, toute une série d'événements peut avoir lieu et venir différer ou empêcher la réalisation de ce procès. L'époque future se définit par conséquent non pas comme l'époque de l'irrévocabilité des faits, mais bien comme un champ de possibles.

$\mathrm{Du}$ point de vue de l'expression linguistique du futur, le futur synthétique à valeur temporelle tend à être remplacé depuis le début $\mathrm{du} \mathrm{XX}^{\mathrm{e}}$ siècle par une périphrase itive (Hagège, 1993), composée d'un verbe de mouvement (aller en français) au présent et de l'infinitif à valeur d'intention. Comme le montre Fleischman (1982: 80), ces périphrases sont le reflet d'un « moving-ego ", c'est-à-dire d'un sujet en action. On peut donc réaliser la synthèse suivante sur les liens entre conception de l'avenir, notion épistémique d'incertitude et expression linguistique du futur : 


\begin{tabular}{|c|c|c|c|}
\hline & $\begin{array}{l}\text { Conception } \\
\text { Mythique }\end{array}$ & Conception Cognitive & $\begin{array}{l}\text { Conception } \\
\text { Phénoménologique }\end{array}$ \\
\hline Époque & $\begin{array}{l}\text { Antiquité }>\text { St } \\
\text { Augustin }\end{array}$ & \begin{tabular}{|l|l|} 
St $\quad$ Augustin $\quad>$ \\
Positivisme
\end{tabular} & Période Contemporaine \\
\hline $\begin{array}{l}\text { Conception } d u \\
\text { temps }\end{array}$ & Cyclique & Linéaire & Ramifiée \\
\hline $\begin{array}{l}\text { Conception } \mathrm{du} \\
\text { futur }\end{array}$ & \begin{tabular}{|lr} 
L'avenir & est \\
inconnu & mais \\
inévitable &
\end{tabular} & $\begin{array}{llr}\text { L'avenir } & \text { est } & \text { prévisible } \\
\text { dans } & \text { un } & \text { cadre } \\
\text { régulateur. } & \end{array}$ & $\begin{array}{l}\text { L'avenir est l'expression de la } \\
\text { liberté de l'Homme }\end{array}$ \\
\hline $\begin{array}{l}\text { Conception } \mathrm{du} \\
\text { possible }\end{array}$ & $\begin{array}{l}\text { Il n'y a pas de } \\
\text { possible }\end{array}$ & $\begin{array}{l}\text { Le possible est ce qui est } \\
\text { réalisable }\end{array}$ & $\begin{array}{l}\text { Le possible est ce qui inscrit la } \\
\text { puissance de l'Homme }\end{array}$ \\
\hline $\begin{array}{l}\text { Expression } d u \\
\text { futur }\end{array}$ & habere + infinitif & futur synthétique & aller + infinitif \\
\hline $\begin{array}{l}\text { Valeur du } \\
\text { temps employé }\end{array}$ & obligation & projection dans le temps & intention \\
\hline
\end{tabular}

Tableau 1

29 Après avoir mis en évidence les liens possibles entre conception ontologique et expression linguistique du futur, on se propose de décrire le système de modélisation des temps verbaux dans le cadre duquel est développée la valeur en langue des formes de futur.

\section{Système de modélisation des temps verbaux}

\subsection{Production de sens et processus d'actualisation}

L'analyse que nous entendons mener s'inscrit dans les cadres d'une linguistique de l'actualisation (Cf. Barberis, Bres, Siblot, 1998) et d'une linguistique du signifiant (Cf. Chevalier, Launay, Molho, 1984) menées dans une perspective monosémiste.

On distingue le plan de la langue (qui fournit les différents morphèmes, i.e. des outils de production de sens dotés chacun d'un signifié de langue ayant une valeur particulière), et le plan du discours (où se situent les référents, à savoir les divers effets de sens produits). Le passage de la langue au discours se fait par le biais de l'actualisation i.e. le processus au cours duquel les signifiés des signes en présence dans l'énoncé se combinent en interaction entre eux et avec le contexte. Dans la diversité des sens produits résultativement, le temps verbal donne toujours les mêmes instructions, celles qui procèdent de sa valeur en langue. La pluralité observable au niveau discursif est le résultat de l'interaction des mêmes instructions du temps verbal avec des éléments co (n)textuels différents. C'est ce que nous prouve l'analyse de l'exemple suivant au futur simple :

(1) Ce sera sans doute Mayotte (titre, blog Le Jardin du Grillon, 14/03/2010) 
Cet énoncé contient une structure généralement typique de celles dans lesquelles le futur a un sens conjectural (du type "On sonne à la porte. Ce sera le facteur ») mais peut être interprété au moins de deux façons :

(1a) Un DOM a eu un rôle déterminant dans l'élection de Sarkozy. Ce sera sans doute Mayotte.

(1b) Quant au grand départ, il se confirme que nous ne rejoindrons pas Manihi [...]

Ce sera donc sans doute Mayotte.

Dans (1a), l'énoncé au futur a un sens modal et exprime une conjecture. Il est l'équivalent de l'énoncé « ce doit être Mayotte ». En (1b), l'énoncé a un sens temporel. Dans l'exemple authentique relevé, l'énoncé avec un contexte plus large était le (1b) et avait donc un sens temporel, en dépit des deux éléments (la structure en « ce sera » et le «sans doute ») qui orientaient plutôt vers une interprétation modale épistémique a priori. Cet exemple confirme bien ce qu'établit la linguistique de l'actualisation: aucun signe ne porte l'entière responsabilité de la production de sens, et que celle-ci doit être entendue comme le résultat de l'opération combinatoire qu'est l'actualisation et non comme l'héritière d'un signifié particulier.

\subsection{Un système élaboré à partir de Reichenbach (1947)}

Le système proposé est issu d'un travail qui a été présenté dans 2 travaux (Azzopardi, Bres, 2016, Azzopardi, Bres 2017) et est le fruit d'une réflexion sur la description des temps verbaux de façon plus large que celle menée sur le futur. Il est basé sur celui de Reichenbach (1947) auquel on apporte certaines modifications.

Le système reichenbachien se signale par sa grande économie : trois points : $\mathrm{E}$ (event point, le procès), $\mathrm{R}$ (reference point, le point de référence) et $\mathrm{S}$ (speech point, le point de l'énonciation) (même si le premier est parfois non un point mais un « segment »), et deux relations : l'antériorité et la coïncidence. Cependant, ce système ne permet pas de rendre compte de l'ensemble des temps verbaux de l'indicatif dans les langues romanes et de leurs emplois en discours. C'est par exemple le cas de l'imparfait : le schéma laisse entendre que $\mathrm{R}$, en relation de coïncidence avec E, est également un segment temporel, alors que, en accord avec la grande majorité des approches, l'imparfait de « je voyais Jean " sera analysé comme représentant l'événement dans son cours à partir d'un point de référence. Soit donc une relation d'inclusion de $\mathrm{R}$ dans $\mathrm{E}$, qui n'est pas analysable à partir des seules notions d'antériorité et de coïncidence.

Notre approche reprend le système de Reichenbach et ses paramètres en y apportant certains ajouts et rectifications.

\subsection{Définitions des paramètres en jeu}

7 Tout comme celui de Reichenbach, le système proposé fonctionne à partir de 3 paramètres que nous définissons de la sorte :

- Le paramètre de l'énonciation S (et le cas échéant S') est un point ;

- Les paramètres du procès $\mathrm{E}$ et, le cas échéant, de l'auxiliaire $\varepsilon$, sont des intervalles : $\mathrm{E}_{\mathrm{i}}-\mathrm{E}_{\mathrm{t}}$, $\varepsilon_{\mathrm{i}}-\varepsilon_{\mathrm{t}}$; l'élément $\mathrm{E}$ étant un procès, il peut être représenté dans sa phase processuelle $\left(\mathrm{E}_{\mathrm{i}}-\mathrm{E}_{\mathrm{t}}\right.$ ), mais également dans sa phase pré-processuelle $\left(\mathrm{E}_{\mathrm{i}}^{\text {prep }}-E^{\text {prep }}\right)$ ou dans sa phase postprocessuelle $\left(E^{\text {postp }}{ }_{i}-E^{\text {postp }}\right)$. À cela servent les auxiliaires, qui disposent d'un intervalle 
noté $\left(\varepsilon_{\mathrm{i}}-\varepsilon_{\mathrm{t}}\right)$ et tout comme la phase processuelle $\mathrm{E}_{\mathrm{i}}-\mathrm{E}_{\mathrm{t}}$ du verbe dispose d'un paramètre de la référence $R$, la phase processuelle $\varepsilon_{\mathrm{i}}-\varepsilon_{\mathrm{t}}$ des auxiliaires dispose d'un paramètre de la référence $\rho$;

40 - Les paramètres de la référence $R$ et $\rho$ sont des points ou des intervalles selon le type de relation (inclusion, coïncidence, neutralité) qu'ils entretiennent avec les paramètre $\mathrm{E}$ et $\varepsilon$ ;

41 - aux relations d'antériorité $(-)$ et de coïncidence $(=)$, nous ajoutons les relations d'inclusion $(\subset)$, e neutralité (ㄷ) et d'antériorité proche (<).

\subsection{Fonctionnement du système}

42 - Les temps simples prennent en charge la représentation de la phase processuelle. La relation $\mathrm{E} / \mathrm{R}$ détermine la représentation aspectuelle; la relation $\mathrm{S} / \mathrm{R}$, la localisation temporelle.

43 - Dans les formes composées, le participe passé (dorénavant "pp.») du verbe saisit l'intervalle $\mathrm{E}_{\mathrm{i}}-\mathrm{E}_{\mathrm{t}}$ sur sa borne terminale : $\mathrm{R}=\mathrm{Et}$. L'auxiliaire donne une instruction aspectuelle (relation entre $\rho$ et $\varepsilon_{\mathrm{i}}-\varepsilon_{\mathrm{t}}$ ) et une instruction temporelle (relation entre $\rho$ et $\mathrm{S}$ ). $\rightarrow$ aux + V. au pp. : Le procès est actualisé dans sa phase post-processuelle, localisée temporellement via la médiation du point de référence $\rho$ de l'auxiliaire.

44 - Dans les formes périphrastiques, l'infinitif du verbe saisit l'intervalle $\mathrm{E}_{\mathrm{i}}-\mathrm{E}_{\mathrm{t}}$ dans sa globalité réduite à un point : le point de référence coïncide avec la phase processuelle : $\mathrm{R}=\mathrm{E}$. L'auxiliaire donne une instruction aspectuelle et une instruction temporelle. Le sémantisme de l'auxiliaire indique la phase de E qui sera saisie par la forme périphrastique.

45 C'est dans ces cadres théoriques qu'on se propose de définir la valeur du signifié de langue des formes de futur actuelles: futur simple, futur antérieur et futur périphrastique, ces valeurs étant basées sur leur morphologie et leurs emplois en discours.

46 Ce système permet une description plus fine des temps verbaux de l'indicatif que les précédents systèmes. En effet, il tient compte de nouveaux paramètres $\left(S^{\prime}, \mathcal{E}\right.$ et $R$ et $\rho$ ) et d'un plus grand type de relations entre ces paramètres (relations d'inclusion $(\subset)$, e neutralité ( $\subseteq$ ) et d'antériorité proche (<)). Cela permet dans le cadre de l'analyse du futur d'inclure l'analyse des formes périphrastiques avec lesquelles les formes synthétiques entrent en concurrence sur la base de paramètres communs.

\section{Description de la valeur du signifié de langue du futur}

\subsection{Valeur du signifié de langue du futur simple}

(2) Guillaume partira en vacances

Formule $: \mathrm{R} \subseteq \mathrm{E}_{\mathrm{i}}-\mathrm{E}_{\mathrm{t}} ; \mathrm{S}-\mathrm{R}$

48 Le procès partir est saisi dans sa phase processuelle, globalement ou cursivement selon le co(n)texte (représentation aspectuelle sous-déterminée, $\left.R \subseteq E_{i}-E_{t}\right)$. Cette saisie est située 
dans l'époque future (localisation temporelle dans laquelle l'énonciation est antérieure au point de référence, $\mathrm{S}-\mathrm{R}$ ).

Conséquence du point de vue des modalités : l'événement est envisagé comme non réalisé et encore réalisable. Cette valeur peut être schématisée au moyen de la figure suivante :

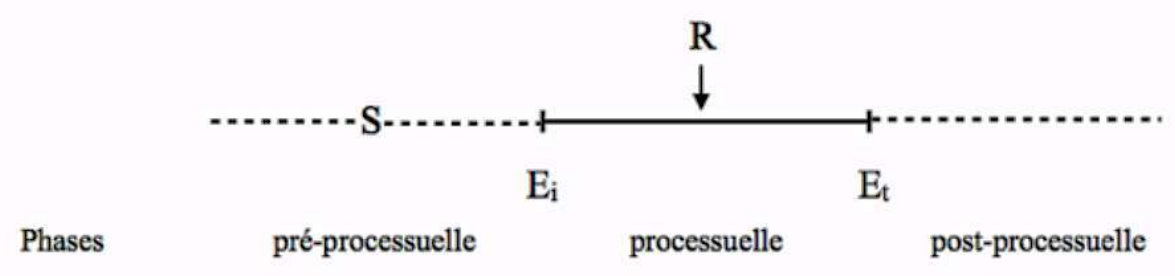

Figure 1

\subsection{Valeur du signifié de langue du futur antérieur}

(3) Guillaume sera parti en vacances

Formule : $\rho \subseteq \mathrm{E}_{\mathrm{i}}^{\text {postp }}$ - $\mathrm{E}_{\mathrm{t}}^{\text {postp }} ; \mathrm{S}-\rho$

Le futur antérieur, combinaison d'un auxiliaire au futur et du V. au pp., saisit le procès dans sa phase post-processuelle, globalement ou cursivement selon le co(n)texte (représentation aspectuelle sous- déterminée, $\rho \subseteq \mathrm{E}_{{ }_{\mathrm{i}}^{\text {postp }}}$ - $\mathrm{E}^{\text {postp }}{ }_{\mathrm{t}}$ ). Cette saisie est située dans l'époque future (localisation temporelle dans laquelle l'énonciation principale est antérieure au point de référence, $S-\rho$ ). Cette valeur peut être schématisée au moyen de la figure suivante :

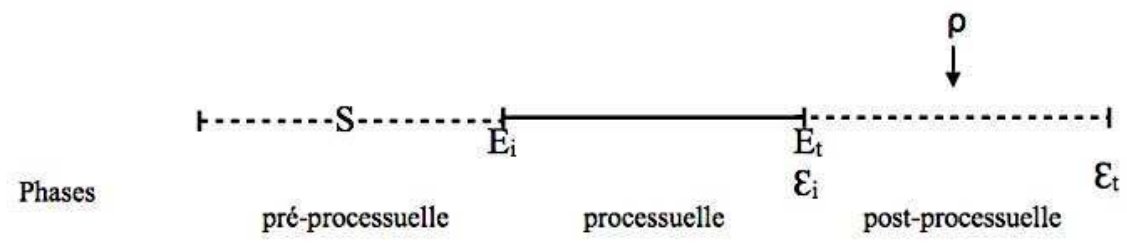

Figure 2

\subsection{Valeur du signifié de langue du futur périphrastique}

51 Aller, en tant que verbe, signifie le mouvement vers un point donné. Grammaticalisé en auxiliaire, il indique le mouvement vers la phase processuelle du procès dénoté et permet de le saisir dans sa phase pré-processuelle, à proximité de sa phase processuelle.

(4) Guillaume va partir en vacances

Formule $: \rho<\mathrm{E}_{\mathrm{i}}-\mathrm{E}_{\mathrm{t}} ; \rho=\mathrm{S}$

Le futur périphrastique, combinaison de l'auxiliaire aller au présent et du V. à l'infinitif, saisit le procès dans sa phase pré-processuelle, en un point de référence à proximité de la phase processuelle du procès $\left(\rho<E_{i}-E_{t}\right)$. Cette saisie située dans l'époque présente (localisation temporelle dans laquelle le point de référence $\rho$ coïncide avec l'énonciation principale $S, \rho=S$ ). Cette valeur peut être schématisée au moyen de la figure suivante : 


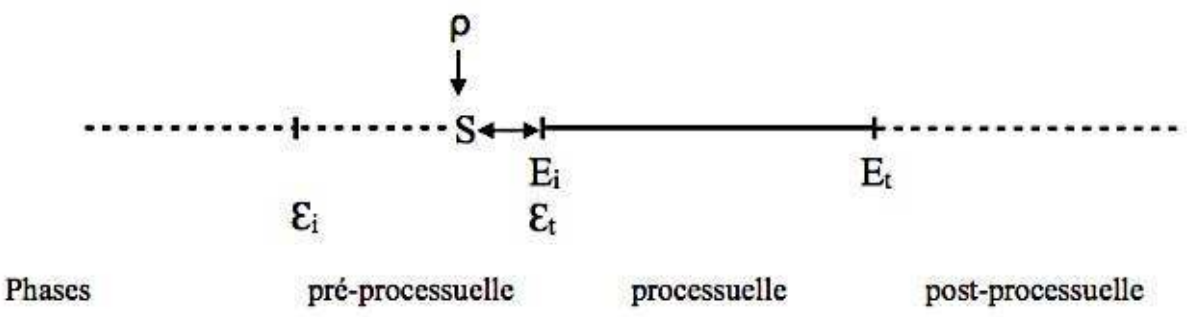

Figure 3

C'est à partir de ces valeurs en langue que l'on entend analyser les différents emplois de chacune des formes de futur en discours. Il ne s'agira pas ici de recenser ni d'analyser l'ensemble des emplois possibles de chacune des formes mais plutôt d'analyser 3 types d'énoncés (un énoncé temporel au futur simple, un énoncé modal (conjectural) au futur simple et un énoncé temporel au futur périphrastique) au cœur des deux grandes problématiques du futur qui ont été évoquées en introduction: le fait que le futur se trouve au croisement entre temporalité et modalité et le fait que futur morphologique et futur périphrastique soient en concurrence dans les énoncés temporels.

\section{Analyse d'emplois en discours}

\subsection{Analyse d'un énoncé temporel au futur simple} globalement ou cursivement selon le contexte. Dans cet énoncé, le procès annoncer dénote un achèvement. En effet :

« La SNCF a annoncé la nouvelle offre TGV en 10 minutes »

- il est incompatible avec arrêter de:

?? «La SNCF a arrêté d'annoncer la nouvelle offre TGV à cause d'un problème »

De ce fait, dans (5), le procès est saisi globalement, c'est-à-dire de sa borne initiale à sa borne terminale.

On a donc $\mathrm{R}=\mathrm{E}_{\mathrm{i}}-\mathrm{E}_{\mathrm{t}}$

Du point de vue temporel, le procès dénoté par le verbe annoncer au futur est situé dans l'ultériorité par rapport au moment de l'énonciation principale. Plusieurs éléments concourent à produire cette interprétation temporelle : la date à laquelle l'énoncé a été produit, le circonstanciel « fin janvier » et le futur simple.

63 Le futur simple saisit le procès dans l'ultériorité par rapport au point de l'énonciation principale S. L'énoncé a été produit le 10 janvier 2017, date qui marque le point de 
l'énonciation principale S. Par rapport à S, le circonstanciel « fin janvier » est situé dans l'ultériorité et sert de point de repère pour la phase processuelle de annoncer. Par conséquent, les éléments co(n)textuels ${ }^{2}$ ont les mêmes instructions que la valeur en langue du futur simple, ce qui conduit à une interprétation temporelle de cet énoncé. En l'absence d'éléments co(n)textuels indiquant un degré de certitude élevé, la réalisation du procès comporte un degré d'incertitude inhérent à la majorité des procès situés dans l'avenir. La saisie du procès dans cet énoncé peut donc être schématisée de la façon suivante :

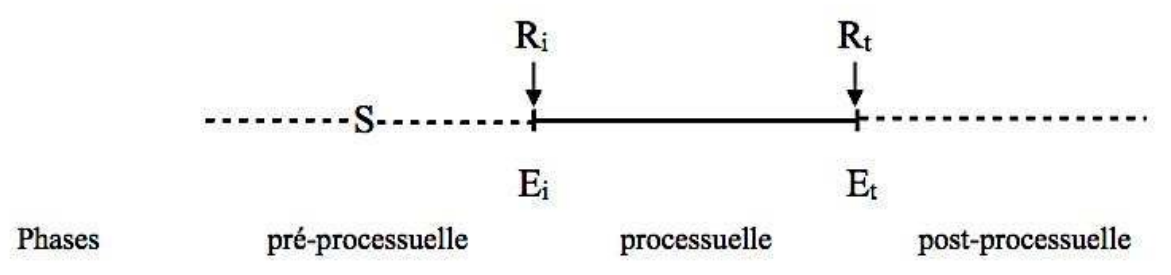

Figure 4

Cette analyse met en évidence deux choses : d'une part, le fait que la formule proposée pour la description de la valeur en langue du futur simple permet de rendre compte de ce type d'emploi, et d'autre part que le sens temporel de l'énoncé n'est pas imputable seulement au temps verbal mais est le produit de l'interaction des différents éléments de l'énoncé entre eux et avec le contexte. L'énoncé (5) est représentatif de l'emploi prototypique du futur simple et il n'y avait donc a priori aucune difficulté à son analyse au moyen de la modélisation proposée. Afin de mettre à l'épreuve cette modélisation, nous allons procéder à l'analyse d'un énoncé à sens conjectural au futur simple également.

\subsection{Analyse d'un énoncé modal au futur simple}

Soit l'énoncé (6) au futur simple :

(6) Ce sera sans doute le site le plus partagé aujourd'hui sur Facebook (extrait d'un post sur Facebook, 21/09/2016)

Du point de vue aspectuel, le futur simple saisit la phase processuelle du procès globalement ou cursivement selon le contexte. Dans cet énoncé, le procès dénote un état étant donné qu'il est exprimé par le verbe être. Le procès est donc saisi cursivement. On a donc la formule suivante :

$$
\mathrm{R} \subseteq \mathrm{E}_{\mathrm{i}}-\mathrm{E}_{\mathrm{t}}
$$

Du point de vue temporel, le procès dénoté par le verbe être au futur simple est situé dans le présent du moment de l'énonciation principale. En effet, l'adverbe temporel « aujourd'hui » situe le procès dans le présent. On a donc:

$\mathrm{S}=\mathrm{R}$

70 Cette indication est contradictoire avec l'instruction temporelle de la valeur en langue du futur simple qui situe quant à elle la phase processuelle du procès dans l'ultériorité par rapport à $\mathrm{S}(\mathrm{S}-\mathrm{R})$. De ce fait, l'instruction temporelle du futur simple ne peut s'appliquer dans cet énoncé et n'est donc pas actualisée en discours. 
71 Du point de vue modal, le procès est envisagé comme non réalisé, et comme réalisable. Il comporte donc un certain degré d'incertitude inhérent à cette non réalisation. Comme cette incertitude s'applique à un procès situé dans le présent, cette instruction vient renforcer le fait que ce procès est une hypothèse formulée par le locuteur.

L'effet de sens produit est donc celui d'une conjecture (hypothèse destinée à expliquer un phénomène surprenant) dont le degré de certitude n'est pas total. La saisie du procès par le futur dans cet énoncé peut être schématisée de la façon suivante :

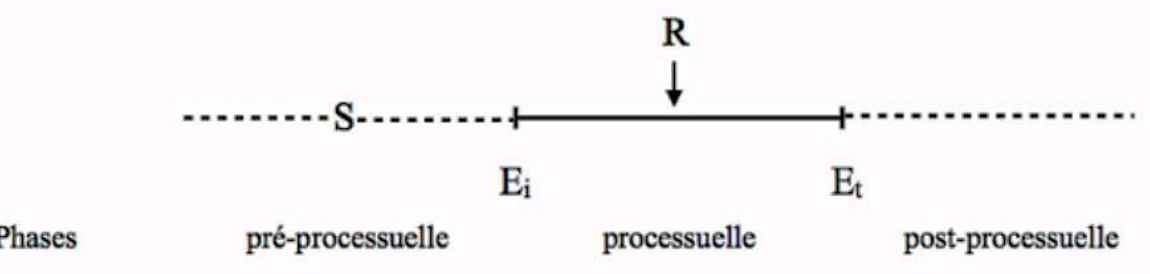

Figure 5

Mais la saisie temporelle du procès dans l'énoncé est celle-ci :

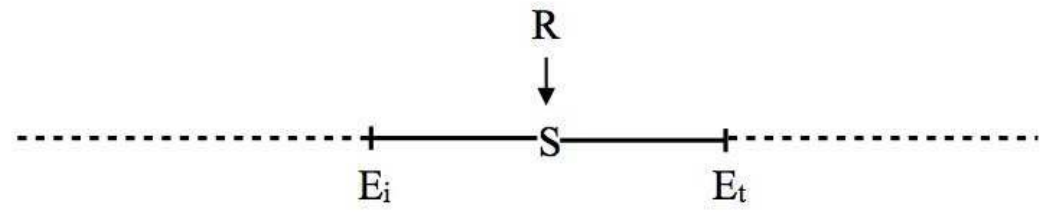

Phases pré-processuelle processuelle post-processuelle

Figure 6

Comme lors de l'analyse de l'énoncé (5), on a pu ici mettre en évidence non seulement que la modélisation de la valeur en langue du futur simple présentée dans le cadre de ce travail permet de rendre compte d'emplois complexes comme l'emploi conjectural, mais aussi que le futur simple n'est pas le seul élément responsable de cet effet de sens modal. Ce système s'écarte des analyses traditionnelles dites de "vérification future " qui expliquent la production de l'effet de sens conjectural comme le placement dans le futur du moment où aura lieu la vérification de l'hypothèse formulée dans le présent.

L'analyse proposée dans ce travail permet de décrire le mécanisme d'interaction de la valeur en langue des différents éléments de l'énoncé entre eux et avec le contexte dont résulte l'effet de sens conjectural.

\subsection{Analyse d'un énoncé temporel au futur périphrastique}

76 Rappelons ici la formule permettant de modéliser la valeur en langue du futur périphrastique :

$$
\rho<\mathrm{E}_{\mathrm{i}}-\mathrm{E}_{\mathrm{t}} ; \rho=\mathrm{S}
$$

77 Soit l'énoncé (7) au futur périphrastique :

(7) Le méga-contrat du RER va donner de l'air aux usagers et à Alstom (Libération, 11/01/2017) 

processuelle, en un point de référence à proximité de la phase processuelle du procès. On a donc la formule suivante :

$$
\rho<\mathrm{E}_{\mathrm{i}}-\mathrm{E}_{\mathrm{t}}
$$

Du point de vue temporel, la phase pré-processuelle est saisie au moment de l'énonciation principale $\mathrm{S}$. Du point de vue modal, la phase pré-processuelle du procès est envisagée comme réalisée, et sa phase processuelle comme réalisable. La saisie du procès dans cet énoncé peut être schématisée au moyen de la figure suivante :

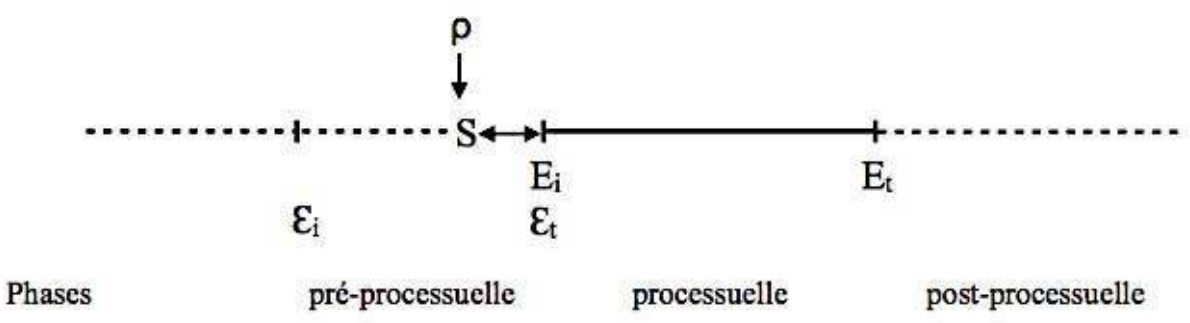

Figure 7

Quelle différence peut-on établir entre futur simple et futur périphrastique dans un énoncé temporel comme celui-ci ? Elle se situe à deux niveaux : d'une part, du point de vue de la localisation du point de repère, d'autre part du point de vue du degré de certitude avec lequel le procès peut être envisagé. Avec le futur simple, le paramètre $R$ qui saisit le procès est situé dans l'avenir, au moment où le procès est censé se réaliser. Avec le futur périphrastique, le paramètre $\rho$ est quant à lui situé dans le présent, au moment de l'énonciation principale $S$. De ce fait, l'ancrage dans la réalité du locuteur est plus important avec le futur périphrastique qu'avec le futur simple. De ce fait, avec le futur périphrastique, de par cet ancrage dans le présent et de par le sémantisme du verbe de mouvement, on a affaire à l'expression d'une action de la part du sujet qui est de réaliser ou de voir se réaliser la phase préparatoire d'un procès à venir. C'est cette différence d'ancrage du point de saisie qui peut avoir pour conséquence une différence de degré de certitude dans la réalisation du procès. En effet, si la phase préparatoire du procès est réalisée, il a plus de chances d'avoir lieu que dans le cas où cette phase préparatoire n'est pas envisagée.

81 Cette différence de perspective entre le futur périphrastique qui met en scène un sujet qui agit et le futur simple qui montre la réalisation d'un procès dans l'avenir sans prendre en compte d'action de la part du sujet est clairement visible dans la suite de l'énoncé (7) :

(8) Le Stif et la SNCF vont passer commande de 255 rames destinées aux RER D et E au consortium Alstom-Bombardier pour un montant total de 3,7 milliards d'euros. Ce « RER du futur » permettra de soulager le réseau et bénéficiera surtout au groupe français. (Libération, 11/01/2017)

Il va de soi que dans cet énoncé, qui constitue le début de l'article dont (7) est le titre, les procès " passer ", " permettre » et " bénéficier » sont situés dans l'ultériorité par rapport au point de l'énonciation principale $S$ qui se trouve être le 11/01/2017. On pourrait penser de prime abord que le procès au futur périphrastique est antérieur aux procès au futur simple : en effet, du point de vue logique, il faut d'abord que le Stif et la SNCF aient passé commande de 255 rames pour que cela puisse dans un second temps soulager le réseau et que le groupe puisse en retirer des bénéfices. Cependant, le procès « donner de 
l'air » de l'énoncé (7) se situe lui aussi au même moment que le procès "permettre (de soulager) ». Il est pourtant conjugué au futur périphrastique.

Il semble donc évident que la différence entre les deux formes de futur n'est pas fonction de la localisation temporelle objective de la réalisation du procès. La différence se situe bien dans le point de vue adopté sur la réalisation de ce procès et le rôle que le sujet peut y jouer. On peut voir si l'on compare les figures 5 et 6 que dans les 2 cas, la réalisation du procès est située au même moment. Comme cela a été souligné, la différence réside dans la localisation du point de saisie du procès: dans le futur pour le futur simple (figure 5), dans le présent pour le futur périphrastique (figure 6). Avec le futur périphrastique, ce qui est dénoté, c'est le sujet agissant : le sujet qui voit ou met en œuvre la réalisation de la phase pré-processuelle du procès. C'est bien le cas dans les énoncés (7) et (8) : le fait de donner de l'air aux usagers et de passer commande implique bien une action de la part du sujet. Au contraire, le fait de permettre de soulager le réseau et de bénéficier de cette opération renvoie à des procès pour lesquels le sujet est passif.

Cette différence est visible dans la valeur en langue des temps verbaux employés et on pourrait mettre en relation la fréquence d'emploi croissante du futur périphrastique depuis le début du XXe siècle avec la conception phénoménologique du temps et avec celle de l'avenir comme expression de la puissance et de la liberté de l'Homme. Cette analyse, à la croisée des regards philosophique et linguistique sur ce phénomène, doit être étayée par une analyse systématique sur un corpus plus vaste et fera l'objet d'un prochain travail de recherche.

\section{Conclusion}

Ce travail a permis de proposer un système de modélisation qui dérive la valeur en langue des temps du futur de leur morphologie mais aussi de l'histoire de leur formation en tenant compte de l'évolution des conceptions de l'avenir dans les cultures étudiées.

Grâce à l'analyse qui a été menée, on a pu mettre en évidence que ce système permet de rendre compte de façon unifiée de la valeur en langue des différentes formes du système verbal : formes simples, formes composées et périphrastiques. Du point de vue de l'étude du futur en particulier, la modélisation de la valeur en langue proposée pour chacune des formes de futur (futur simple, le futur antérieur et le futur périphrastique) permet de rendre compte des différents emplois de ces formes, qu'ils soient temporels ou modaux. Elle permet également de développer certaines pistes de réflexion en vue d'une meilleure compréhension de la concurrence entre futur simple et futur périphrastique au-delà des traditionnels arguments qui touchent à la proximité temporelle et qui rencontrent un certain nombre de contre-exemples (à ce sujet, on renvoie notamment à Abouda, Skrovec, 2015).

87 Certaines pistes de recherche sont encore à explorer. Il serait notamment intéressant dans la perspective qui est celle de ce volume de voir dans quelle mesure ce système, qui a été par ailleurs testé sur le français et l'espagnol, s'applique aux autres langues romanes et aux divers emplois temporels et modaux des temps du futur dans ces langues. 


\section{BIBLIOGRAPHIE}

ABOUDA, L., AZZOPARDI, S., SKROVEC, M., 2016, « Le Futur », Fiche de grammaire du français vernaculaire, projet FRACOV, <http://www.univ-paris3.fr/index-des-fiches-227311.kjsp>

ABOUDA, L., SKROVEC, M., 2015, « Du rapport entre formes synthétique et analytique du futur. Étude de la variable modale dans un corpus oral micro-diachronique ", RSP, 38, Le Futur, Presses Universitaires d'Orléans, Orléans, p. 35-58.

ALVAREZ CASTRO, C., 2007, «Interprétation du futur de l'indicatif et représentations d'événements futurs », Cahiers Chronos, 19, p. 7-24.

AZZOPARDI, S., 2011, Le futur et le conditionnel : valeur en langue et effets de sens en discours. Analyse contrastive espagnol / français, Montpellier, Université de Montpellier 3 : thèse de doctorat, Sciences du langage, sous la direction de Bres, Jacques. <http://www.biu-montpellier.fr/ florabium/jsp/nnt.jsp?nnt=2011MON30052>

AZZOPARDI, S., BRES, J., 2017, « Le système temporel et aspectuel des temps verbaux de l'indicatif (en français) ", Apothéloz, D., Vetters, C. (coord), Quand les temps verbaux français font système, Revue Verbum, XXXIX (1), Presses Universitaires de Nancy, Nancy, p. 71-112.

AZZOPARDI, S., BRES, J., 2016, « Revisiter Reichenbach ? Pour une approche sémantique systématique des temps verbaux de l'indicatif (en français) ", Actes du $5^{e}$ Congrès Mondial de Linguistique Française 2016, <http://dx.doi.org/10.1051/shsconf/20162712002>.

BARANZINI, L., 2017, Le futur dans les langues romanes, Bern, Bruxelles, Frankfurt am Main, New York, Oxford, Warszawa, Wien : Peter Lang.

BARBERIS, J-M., BRES, J., SIBLOT, P. (éds), 1998, De l'actualisation, CNRS-Editions, Paris.

BARCELO, G. J., 2007, « Le futur des langues romanes et la modalité : monosémie et dialogisme », Cahiers de praxématique, 47, Aspectualité, temporalité, modalité, p.177-190.

BAUHR, G., 1992, « Sobre el futuro cantaré y la forma compuesta voy a cantar en español moderno ", M Sprâk, 86/1, p. 69-79.

BERGER, G., 1964, Phénoménologie du temps et prospective, Presses Universitaires de France, Paris.

BOSKOVIC, S., 2006, «Le temps et l'espace : de la conscience mythique à la conscience phénoménologique » Cahiers du Mimmoc, 2, < http:// mimmoc.revues.org/204>.

BRES, J., 2009, « La marquise sortira à cinq heures... Futur de l'indicatif en français et textualité narrative », Faits de Langues, 33, Le Futur, p. 197-210.

BRES, J., LABEAU, E., 2012, « Un phénix linguistique ? Le tour narratif va + infinitif renaîtrait-il, en français contemporain, de ses cendres médiévales? ", dans C. Guillot et al. (éds), Le Changement en français, Berne, Peter Lang, p. 1-14.

BYBEE, J., PERKINS, R. \& PAGLIUCA, W., 1994, The Evolution of Grammar : Tense, Aspect and Mood in the Languages of the World, University of Chicago Press, Chicago.

CHEVALIER, J-C., LAUNAY, M. \& MOLHO, M., 1984, « La raison du signifiant », Modèles linguistiques, $\mathrm{VI} / 2$, p. 27-41. 
FLEISCHMAN, S., 1982, The Future in Thought and Language. Diachronic Evidence from Romance, Cambridge University Press, Cambridge.

GARDIES, J-L., 1975, La logique du temps, Presses Universitaires de France, Paris.

HAGÈGE, C., 1993, The Language Builder, John Benjamins, Amsterdam.

KRIPKE, S., 1963, « Semantical Considerations on Modal Logic », Acta Phil. Fennica, 16, p. 83-94.

MARTIN, R., 1981, « Le futur linguistique : temps linéaire ou temps ramifié ? », Langages, vol. 15 / 64, p. 81-92.

MORGENSTERN, A., PARISSE, C., SEKALI, M., 2010, « A la source du futur: premières formes verbales dans les productions spontanées de deux enfants français de 18 mois à 3 ans ", Faits de langues, 33, Le Futur, p. 163-176.

PERET, C., 2013, « Construction diachronique des usages scolaires du futur périphrastique », LIDIL , 47, p. 61-80.

REICHENBACH, H., 1947, Elements of Symbolic Logic. Macmillan \& Co, New York.

RESCHER, N., URQUHART, A., 1971, Temporal logic, Springer-Verlag, Wien, New-York.

RICCI, C., 2017, Les emplois modaux du futur et de l'imparfait: analyse contrastive italien-français avec un regard sur la diachronie, Doctorat, Université de Neuchâtel, Neuchâtel.

ROCCI, A., 2000, «L'interprétation épistémique du futur en italien et en français : une analyse procédurale », Cahiers de linguistique française, 22, p. 241-274.

SABEAU-JOUANNET, E., 1973, «L'expression des modalités aspectivo-temporelles et son évolution chez des enfants de deux à quatre ans ", Etudes de Linguistique Appliquée, 9, p. 91-100.

SAINT AUGUSTIN, Confessions, Pellegrin Pierre (éd), 1998, Nathan, Paris.

SILLETTI, A. M., 2009, «L'expression de la futurité dans un corpus de presse franco-italienne », Revue québécoise de linguistique, 33/1, p. 197-207.

SQUARTINI, M., 2004, « La relazione semantica tra Futuro e Condizionale nelle lingue romanze », Revue Romane, 1 / 39, p. 68-96.

VETTERS, C., LIERE, A., 2009, «Quand une périphrase devient temps verbal : le cas d'aller + infinitif ", Faits de Langues, 33, Le Futur, p. 27-36.

\section{NOTES}

1. Extrait de l'émission «L'avenir du futur » du 10/03/1975 diffusé lors de l'émission « Vladimir Jankélévitch»: Le temps, ce je-ne-sais-quoi et ce presque-rien" sur France Culture le 21/04/2014. Consulté en ligne le 13 mars 2015 (http://www.franceculture.fr/emission-lesnouveaux-chemins-de-la-connaissance-vladimir-jankelevitch-14-le-temps-ce-je-ne-sais-quo)

2. Le terme co(n)texte désigne à la fois les éléments du cotexte et du contexte. 


\section{RÉSUMÉS}

Le futur dans les langues romanes est un des temps verbaux qui fait l'objet de recherches sans cesse renouvelées. Cependant, de nombreux problèmes demeurent dès lors qu'il s'agit d'analyser la valeur en langue de ce temps et le lien qu'elle entretient avec ses emplois en discours. L'objectif dans le cadre de cet article est de mener une réflexion d'ordre plus théorique compatible avec les différentes perspectives depuis lesquelles la question du futur peut être abordée. Il s'agira de donner des éléments d'analyse descriptive du signifié de la valeur en langue du futur qui permette, en diachronie et en synchronie : (a) de rendre compte des spécificités de ce temps en termes de temporalité et de modalité ; (b) de rendre compte des différents emplois en discours du futur; (c) d'analyser la concurrence entre futur morphologique et futur périphrastique ; $(\mathrm{d})$ de mettre en évidence les points communs et différences entre les différentes langues romanes.

The future in romance languages is a constant subject of investigation. However, many questions still remain unsolved especially in the analysis of the meaning in language of the future tenses and its links with their various usages in speech. The aim of this paper is to give a philosophical and linguistic description of the meaning in language of each future tense (simple future, anterior future and periphrastic future) in order to show and explain, in a synchronic and in a diachronic perspective as well: (a) the properties of these tenses in temporal, modal and aspectual terms; (b) how the production of the various usages in speech works; (c) the concurrence between simple future and periphrastic future; (d) the similarities and differences between romance languages.

INDEX

Mots-clés : temps futur, romanistique

Keywords : future tense, romance studies

\section{AUTEUR}

\section{SOPHIE AZZOPARDI}

Université Paris Diderot - CLILLAC-ARP EA3967 